\title{
Combined High-Resolution Optical Tweezers and Multicolor Single-Molecule Fluorescence with Automated Single Molecule Assembly Line
}

Cho-Ying Chuang, Matthew Zammit, Miles L Whitmore, and Matthew J Comstock

Department of Physics and Astronomy, Michigan State University, East Lansing, MI 48824, USA

\section{Supporting information}

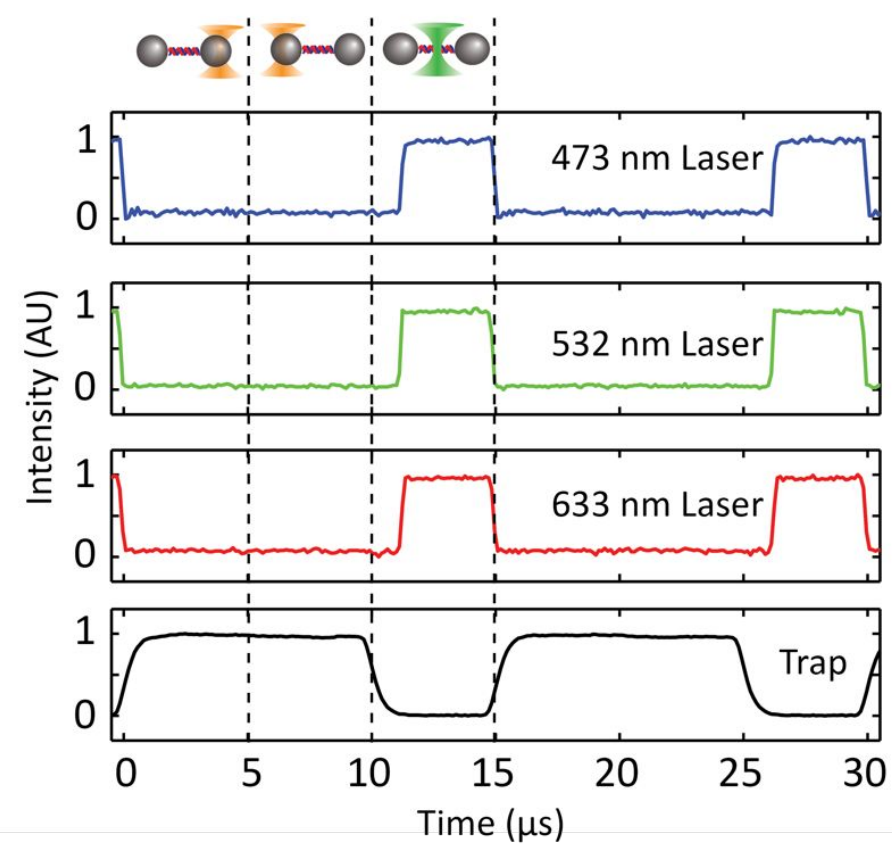

Figure S1. Interlacing and time-sharing of optical traps and fluorescence excitation lasers. Simultaneous measurement of trap and fluorescence excitation laser intensities. The optical traps are turned on and off in sequence during first two time intervals. The fluorescence excitation lasers are only on during the third time interval while the trap laser is off. The interlacing rate is 66 $\mathrm{kHz}(15 \mu \mathrm{s}$ period). Signals were measured by the photodetectors (feedback QPD and PDs) and recorded by a digital oscilloscope.
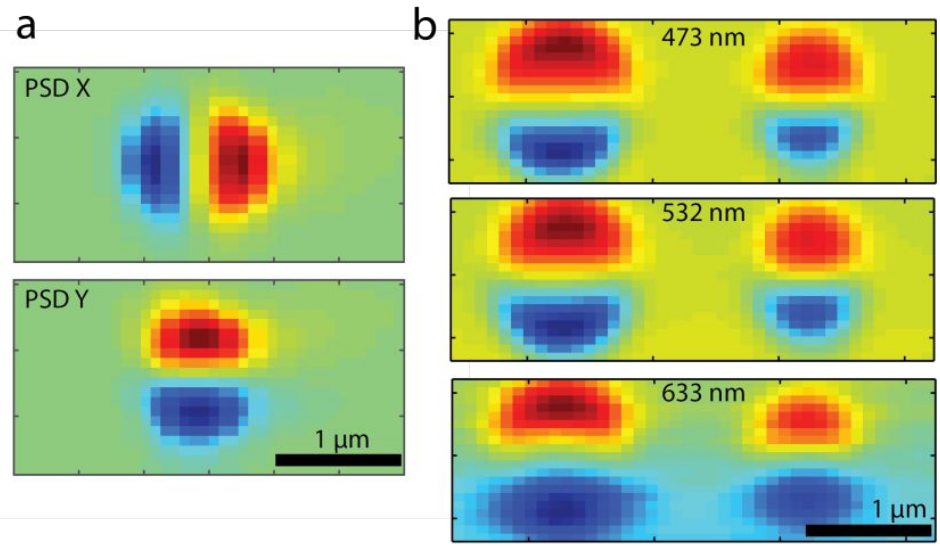

Figure S2. PSD bead detection images from all three fluorescence excitation lasers. Raster scanning the piezo mirror stage positioning the confocal excitation lasers and measuring the bead detection signals using the PSD. A) X (above) and $y$ (below) PSD signal images from scanning the green laser confocal spot over a single trapped bead. The false color red represents positive signal 
and blue represents negative signal. B) PSD y signal from scanning the confocal spot over a pair of trapped beads, and exciting with either the blue (top), green (middle) or red (bottom) excitation lasers. The alignment and image quality are nearly identical.
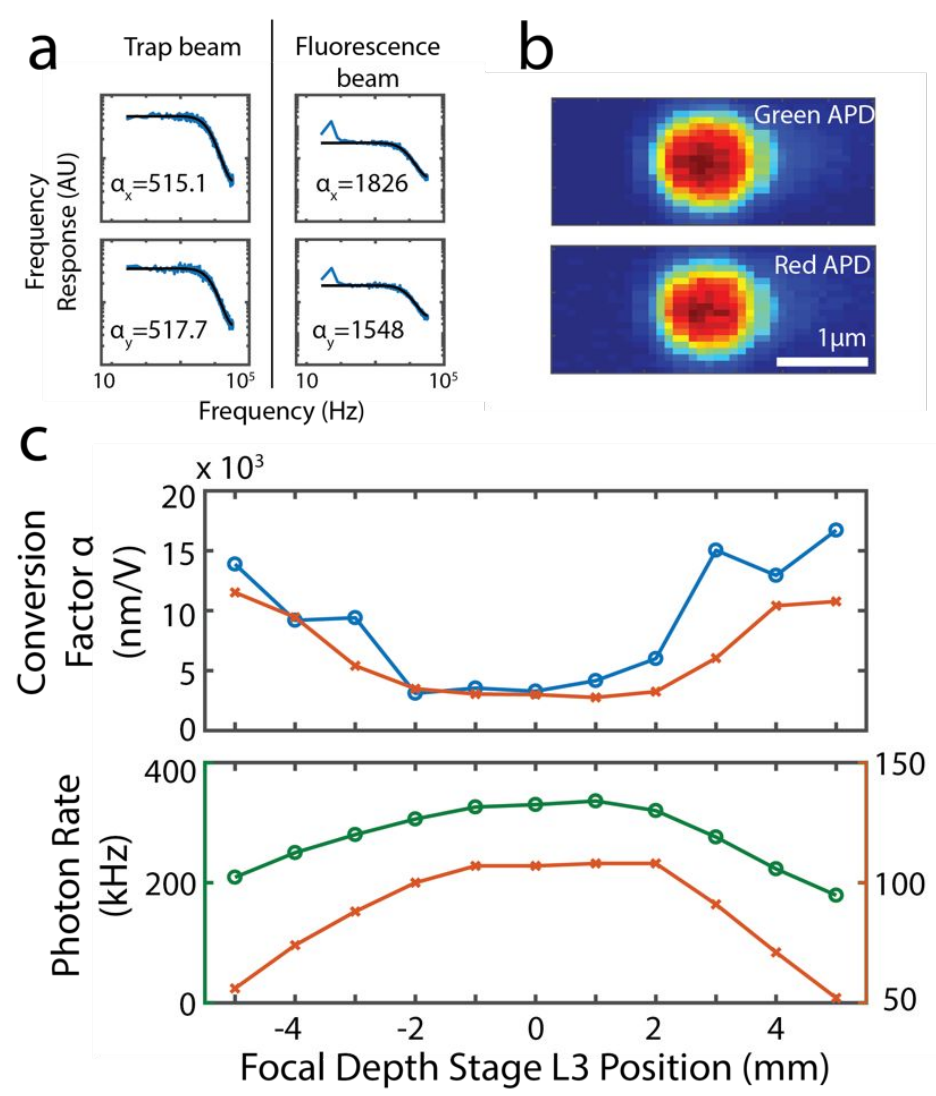

Figure S3. Alignment of confocal spot focal depth. A) Bead position calibration using either trap laser (left) or fluorescence excitation laser (right) in $x$ (upper) and $y$ (lower) direction at the optimal focal depth position. The power spectra are from the same trapped bead. $\alpha \_x$ and $\alpha \_y$ are the position conversion factors (i.e., volt to $\mathrm{nm}$ conversion) found via the standard fitting methods. The values are typical. B) Fluorescence images of a trapped fluorescent bead via raster scanning the piezo mirror stage at the optimal focal depth position. The emission spectrum of the fluorescent bead (F8819, Invitrogen) covers both green (APD 1) and red (APD 2) detection channel wavelengths. C) Adjustment of confocal spot focal depth. (Upper) The conversion factors $\alpha \_x$ (blue circles) and $\alpha \_y$ (red crosses) were determined from the power spectrum fitting in a). (Lower) The fluorescence signals from green APD 1 (green circles) and red APD 2 (red crosses) were obtained from the fluorescence images in b) vs focal depth. The two methods agree on the optimal focal depth. 


\begin{tabular}{|l|l|}
\hline Insert name & Sequence (5' to $\left.3^{\prime}\right)$ \\
\hline hp seq & $\begin{array}{l}\text { /5Phos/ TTG AAA TAC CGA CCG CTC AGC TAT CAG CC/idSp/ CTC TGA CAC } \\
\text { ATG CAG CTC CC }\end{array}$ \\
\hline Probe name & Sequence (5' to $\left.3^{\prime}\right)$ \\
\hline 29mer oligo -bio & GG CTG ATA GCT GAG CGG TCG GTA TTT CAA/3Bio/ \\
\hline
\end{tabular}

Table S1. Complementary hairpin sequence and probe strand for "probe cryptic hairpin binding" construct. Quantum dots are labeled to the probe strands via biotin-streptavidin bonds. Binding sites are shown in bold.

\begin{tabular}{|l|l|}
\hline Insert name & Sequence (5' to ${ }^{\prime}$ ') \\
\hline probe site 1 - 5dT & /5Phos/CCTGG TTTTT AGGACTTGT CCCACTGGC \\
\hline probe site 2 - 5dT & /5Phos/CCTGG TTTTT CAGTATCGA CCCACTGGC \\
\hline 29mer - insert & $\begin{array}{l}\text { /5phos/CCTGG TTT TT GAA ATA CCG ACC GCT CAG CTA TCA GCC } \\
\text { CCCACTGGC }\end{array}$ \\
\hline Probe name & Sequence (5' to 3') \\
\hline probe 1 - Cy3 & /5Phos/ACA AGT CCT /3Cy3Sp/ \\
\hline probe 1 - AF488 & /5Phos/ACA AGT CCT /3AlexF488N/ \\
\hline probe 2 - Cy5 & /5Phos/TCG ATA CTG /3Cy5Sp/ \\
\hline 29mer - AF647 & /5Phos/GGC TGA TAG CTG AGC GGT CGG TAT TTC AA/3AlexF647N/ \\
\hline
\end{tabular}

Table S2. Construct ssDNA insert for synthesizing the "probe hybridization" construct and the complementary fluorophore-labeled probes. Binding sites are shown in bold.

\begin{tabular}{|l|l|}
\hline Insert name & Sequence $\left(5^{\prime}\right.$ to $3^{\prime}$ ) \\
\hline probe site 1 - 5dT - 5dT & /5Phos/ CCT GG TTTTT AGG ACT TGT TTTTT CCC ACT GGC \\
\hline probe sites 1 and 2 & $\begin{array}{l}\text { /5Phos/ CCT GG TTTTT AGG ACT TGT TTTTT CAG TAT CGA TTTTT CCC ACT } \\
\text { GGC }\end{array}$ \\
\hline probe sites 1, 2 and 3 & $\begin{array}{l}\text { /5Phos/ CCT GG TTTTT AGG ACT TGT TTTTT CAG TAT CGA TTTTT ATC GCA } \\
\text { AGT TTTTT CCC ACT GGC }\end{array}$ \\
\hline
\end{tabular}




\begin{tabular}{|l|l|}
\hline Probe name & Sequence (5' to $3^{\prime}$ ) \\
\hline probe 1 - Cy3 & ACA AGT CCT /3Cy3Sp/ \\
\hline probe 1 - Cy3-Cy5 & /5Cy5/ ACA AGT CCT /3Cy3Sp/ \\
\hline probe 1 - AF488 & ACA AGT CCT /3AlexF488N/ \\
\hline probe 2 - Cy5 & TCG ATA CTG /3Cy5Sp/ \\
\hline probe 3 - AF488 & ACT TGC GAT /3AlexF488N/ \\
\hline
\end{tabular}

Table S3. Construct ssDNA insert for synthesizing the "ligated probe" construct and the complementary fluorophore-labeled probes. Binding sites are shown in bold. 
Supplementary Note 1. Detailed list of essential parts for the construction of the instrument shown in Fig. 1.

\section{High-resolution optical tweezers module:}

1064 nm fiber laser (IPG Photonics, YLR-5-1064-LP)

Free-space optical isolator, $1064 \mathrm{~nm}, 4.7 \mathrm{~mm}$ diameter (Thorlabs, IO-5-1064-VHP)

$1 / 2$ wave plate, $1064 \mathrm{~nm}, 0.5$ " (Newport, 05RP02-34)

Polarizing cube beam splitter, high power, 1064 nm, 0.5" (CVI, PBSO-1064-050)

High power beam dump (Kentek, ABD-0.75NP)

AOM1, acousto-optic modulator (IntraAction, ATM-803D16B)

Mirror for reflecting unused AOM1 laser output to power dump, $1064 \mathrm{~nm}$ laser line mirror (Newport, 05D10DM.10)

Lenses for telescopes T1 and T2, BK 7, plano-convex, anti-reflection coated for $1064 \mathrm{~nm}$ laser (e.g., Newport, KPX091AR.33)

M1, $1064 \mathrm{~nm}$ laser line mirror, Zerodur, $\lambda / 20$ (Newport, 10Z40DM.10)

BS1, fused silica broadband beam sampler, $6.10 \mathrm{~mm}$ thick, $\lambda / 10$ flatness, 1010-1550nm (Newport, 10Q20NC.3)

QPD1 \& QPD2, $1064 \mathrm{~nm}$ enhanced quad photodiode (First Sensor, QP154-Q-HVSD)

D1, 1064 nm cutoff short-pass dichroic mirror (Chroma, T970DCSPXR)

O1 \& 02, 60x water-immersion, 1.2 NA microscope objectives (Nikon, CFI Plan APO VC 60XWI)

Sample chamber stage motors (3 each, Newport, CMA-12CCCL) and controller (Newport, ESP300)

D2, 1064 nm cutoff short-pass dichroic mirror (CVI, SWP-45-RP1064-TU780-PW-1012-C)

F3, $1064 \mathrm{~nm}$ laser line filter (Newport, 10LF25-1064)

\section{Bright-field imaging:}

LED (Thorlabs, LED940E)

F1 filter, $1000 \mathrm{~nm}$ cutoff short-pass filter (Thorlabs, FES1000)

F2 filter, $850 \mathrm{~nm}$ cutoff long-pass filter (Thorlabs, FEL850)

IR-enhanced CCD camera (Watec, WAT-902H2)

\section{Multi-color confocal fluorescence spectroscopy module:}

$532 \mathrm{~nm}$ laser (green excitation), diode pumped solid state, $50 \mathrm{~mW}$ (LASOS, GLK 3250 T01)

$633 \mathrm{~nm}$ laser (red excitation), Helium-Neon laser, $17 \mathrm{~mW}$ (Research Electro-Optics)

$473 \mathrm{~nm}$ laser (blue excitation), MLD series diode laser, $80 \mathrm{~mW}$ (Cobolt, 0473-06-01-0080-100) 
AOM2 \& AOM3, acousto-optic modulators (IntraAction, AOM-802AF1, optimized for 532 and $633 \mathrm{~nm}$ respectively, IntraAction ME external controllers)

D10, $659 \mathrm{~nm}$ cutoff short-pass dichroic mirror (Semrock, LM01-659-25)

D9 and D11, 552 nm cutoff short-pass dichroic mirror (Semrock, LM01-552-25)

D8 and D12, $473 \mathrm{~nm}$ cutoff long-pass dichroic mirror (Chroma, ZT473BCM)

$\mathrm{L} 1$, lens to focus all excitation lasers to fiber input aperture, molded aspheric lens, $f=11.0 \mathrm{~mm}, \mathrm{NA}=0.3$ (Thorlabs, A397TM-A)

$L 2$, lens to collimate all excitation lasers from fiber output, achromatic doublet lens, $f=25.4 \mathrm{~mm}$

(Newport, PAC022AR.14)

Single-mode polarization maintaining optical fiber (Thorlabs, TS0986175-PM460-HP)

BS2, pellicle beam splitter, uncoated (Thorlabs, BP108)

PD1, PD2, and PD3, Si photodetector (Thorlabs, PDA36A)

D7, multi-band-pass/multi-laser-reflect dichroic mirror (Chroma, ZT473/532/633RPC-XT)

F8, absorptive neutral density filter (e.g., Thorlabs, NE30A) mounted on remote actuated flip mount (Thorlabs, MFF001)

M2, Piezo mirror stage (Mad City Labs, Nano-MTA2 Invar)

D3, 875 nm cutoff long-pass dichroic mirror (Semrock, FF875-Di01-25-D)

D4, 900 nm cutoff long-pass dichroic mirror (Thorlabs, DMLP900)

PSD, position-sensitive photodetector (First Sensor, DL100-7-PCBA3)

F4 laser line removal filter (Chroma, ZET473/532/635M)

$\mathrm{PH}$, pinhole (Thorlabs or Edmund Industrial Optics, precision pinholes 25-100 um, in precision magnetic removable mount Thorlabs, KB1P)

D5, short-pass dichroic mirror (Chroma, 540DCXR)

F5, Alexa488 emission filter (Chroma, ET510/20M)

D6, short-pass dichroic mirror (Chroma, 640DCXR)

F6, Cy3 emission filter (Chroma, HQ580/60M/2P)

F7, Cy5 emission filter (Chroma, ET685/50M)

APD1, APD2 and APD3, avalanche photodiode single photon counting modules (Excelitas Tech, SPCMAQRH-14). 